\title{
Priming of grasping muscles when viewing a safety handle is diminished with age
}

\author{
D.A.E. Bolton ${ }^{\mathrm{a}, *}$, S.E. Schwartz ${ }^{\mathrm{b}}$, M. Mansourc ${ }^{\mathrm{c}}$, G. Rydalch ${ }^{\mathrm{d}}$, D.W. McDannald ${ }^{\mathrm{a}}$ \\ ${ }^{a}$ Department of Kinesiology \& Health Science, Utah State University, United States \\ ${ }^{\mathrm{b}}$ Department of Psychology, Utah State University, United States \\ ${ }^{c}$ Department of Electrical \& Computer Engineering, Utah State University, United States \\ d Department of Biology, Utah State University, United States
}

\section{A R T I C L E IN F O}

\section{Keywords:}

Motor affordance

Transcranial magnetic stimulation

Reactive balance

Posture

\begin{abstract}
A B S T R A C T
Merely viewing objects within reachable space can activate motor cortical networks and potentiate movement. This holds potential value for smooth interaction with objects in our surroundings, and could offer an advantage for quickly generating targeted hand movements (e.g. grasping a support rail to maintain stability). The present study investigated if viewing a wall-mounted safety handle resulted in automatic activation of motor cortical networks, and if this effect changes with age. Twenty-five young adults (18-30 years) and seventeen older adults (65+ years) were included in this study. Single-pulse, transcranial magnetic stimulation was applied over the motor cortical hand representation of young and older adults shortly after they viewed a safety handle within reaching distance. Between trials, vision was occluded and the environment was unpredictably altered to reveal either a safety handle, or no handle (i.e. covered). Modulation of intrinsic hand muscle activity was evident in young adults when viewing a handle, and this was selective in terms of both the muscles activated and the time at which it emerged. By contrast, older adults failed to show any changes when viewing the safety handle. Specifically, the presence of a handle increased corticospinal activity in hand muscles of young adults when TMS was applied $120 \mathrm{~ms}$ after opening the goggles $(\mathrm{p}=.014)$, but not in the older adults $(\mathrm{p}>.954)$. The fact that the visual priming observed in younger adults was absent in older adults suggests that aging may diminish the ability to quickly put our visual world into automatic motor terms.
\end{abstract}

\section{Introduction}

Simply observing graspable objects within reaching distance can activate motor cortical networks suitable for grasping these objects, suggesting that we put our world into motor terms automatically [1-6]. This effect is evident as faster reaction times when hand responses are subliminally primed [7], but also using measures such as functional Magnetic Resonance Imaging [1] and Transcranial Magnetic Stimulation [6] to detect underlying changes in brain activity when viewing graspable objects. A direct link between visual perception and action offers a way to overcome transmission delays within a distributed nervous system. Consequently, behaviours that must be quick, yet simultaneously goal-directed, stand to become more effective by visually priming potential actions. This extends from the concept of affordances, originally put forth by Gibson which emphasizes complementarity between an animal and it's environment, and the ability to directly perceive opportunities for action [8].
In our laboratory, we recently demonstrated a rapid and selective change in hand muscle activity when young adults viewed a wall-mounted safety handle (i.e. a handle commonly used to aid stability) [9]. These results suggest a rapid engagement of muscles suitable for grasping a safety handle based on vision, and extend upon past affordance work by using a visual stimulus explicitly associated with maintaining stability. Recent evidence indicates that the motor affordance effect is attenuated when participants are faced with a high cognitive load [10], which suggests a link between cognitive resources and visual priming for action. Given the general decline in cognitive function with age [11], the ability to evoke appropriate actions may lessen with age. This may in turn compromise effective interactions with our surroundings (e.g. predispose individuals to fall if they are unable to grasp an available handhold).

The present study was conducted to test if viewing a wall-mounted safety handle results in activation of cortical motor networks in older adults. We hypothesized that the hand area of the primary motor cortex would be facilitated shortly after visual access to a safety handle

\footnotetext{
* Corresponding author at: Department of Kinesiology \& Health Science, Utah State University, 7000 Old Main Hill, Logan, UT 84322-7000, United States.

Email address: dave.bolton@usu.edu (D.A.E. Bolton)
} 
versus when no handle was visible, and that this effect would be diminished in older versus younger adults. These changes in primary motor cortex activity would be revealed through corticospinal excitability (CSE). To address our specific aim, factors established in previous motor affordance research were used to inform a focused design. For example, past studies have shown that objects affording a particular type of grasp produce rapid excitability changes in muscles involved in grasping these viewed objects when they are within graspable range of the individual [2-6] but, this effect was absent when objects were out of reach, and for non-graspable 'control' objects [3]. Based upon this foundation we streamlined our approach to measure CSE in hand muscles during two distinct visual conditions: (i) viewing a wall-mounted safety handle or (ii) viewing no handle (i.e. covered). Here, we extend upon recent findings in our lab where CSE was modulated in young adults when viewing the safety handle to determine if the effect is attenuated in older adults.

\section{Material and methods}

\subsection{Participants}

Twenty-five, healthy, young adults (YA) and seventeen healthy, older adults $(\mathrm{OA})$ provided written informed consent prior to participation. The average ages were $75.4+/-6$ years for the OA group, and $22.8+/-2.9$ years for the YA group. Procedures were approved by the Utah State University, Institutional Review Board conducted in accordance with the Declaration of Helsinki. Participants with neurological illness were excluded from the study. Furthermore, participants were screened prior to testing to assess the suitability for TMS using guidelines developed by a consortium of experts [12]. Note that the YA data are taken from McDannald et al. [9].

\subsection{Protocol}

\subsubsection{Electromyography}

Electromyography (EMG) was recorded using Delsys DE-2.1 differential surface electrodes, and EMG signals were amplified (gain = 1000) using a Delsys Bagnoli-4 amplifier (Delsys Inc., Boston, MA, USA). EMG data was sampled at $5000 \mathrm{~Hz}$ and bandpass filtered using Signal Software and a Cambridge Electronic device (Power 1401, Cambridge Electronic Design, Cambridge, UK). EMG was collected from three intrinsic hand muscles, and a forearm muscle (Extensor Carpi Radialis, ECR) to detect movement onset. The intrinsic hand muscles measured were the First Dorsal Interosseus (FDI), Opponens Pollicus (OP), and Abductor Digiti Minimi (ADM) given the important role of these muscles in gripping objects and past TMS-based studies exploring hand affordance on intrinsic hand muscles $[2-4,6]$.

\subsubsection{Transcranial magnetic stimulation}

Single-pulse TMS was delivered over the hand motor cortical representation while participants completed the experiment. TMS pulses were time-locked to the opening of liquid crystal goggles, and prior to any movement. Magnetic stimuli were delivered to the left primary motor cortex (M1) by a Magstim 200 stimulator using a figure of eight D70 2 Coil (Magstim Company Ltd., Whitland, UK), located at the optimal position to obtain a motor evoked potential (MEP) for the right FDI. Resting motor threshold (RMT) was determined, a stimulator intensity where 5/10 MEPs exceed $50 \mu \mathrm{V}$ peak-to-peak [13]. The test stimuli used throughout the experiment was set at $1.2 \mathrm{x}$ RMT.

\subsubsection{Experimental task}

All testing was conducted with participants seated directly in front of a safety handle mounted on a wall, within comfortable grasping range (Fig. 1A). Throughout testing, participants were told to remain relaxed with their hands resting on their lap while looking at the handle. Access to vision was manipulated in this study by use of liquid crystal goggles (Translucent Technologies Inc. Toronto, ON, Canada). During the visual occlusion period, the handle was covered or uncovered by the experimenter on each trial in a random order, unpredictable to the participant. Participants were instructed to move but only when: (a) the handle was visible (i.e. uncovered) AND (b) if an auditory tone cued a reach to grasp the handle. Each trial started with the occlusion goggles closing for $6 \mathrm{~s}$ after which point the goggles opened to offer a full view. The visual response environment included one of two possible configurations: (a) handle visible (Handle), or (b) handle covered (No-Handle). When the handle was present, participants were required to reach-to-grasp the handle with their right arm as quickly as possible once they heard the tone. TMS pulses were delivered at three different time points ( $80 \mathrm{~ms}, 120 \mathrm{~ms}, 160 \mathrm{~ms}$ ) in separate randomized test blocks. Each block consisted of 45 trials which lasted approximately 10 min per block. Each trial was $10 \mathrm{~s}$ with short pauses before the next trial to allow participants a chance to reset as needed. Participants were given a brief rest period between test blocks. TMS pulses were always delivered after opening the goggles but well in advance of auditory cues (if present). Auditory cues were presented after the goggles opened with an onset delay of either $200 \mathrm{~ms}$ or $1500 \mathrm{~ms}$. For one third of these trials, no auditory cue was presented acting as a 'catch' trial to prevent anticipatory reactions. In addition to the two visual conditions listed above, 'no-vision' reference trials were randomly interspersed throughout collection blocks to deliver TMS without opening the goggles. The purpose of this condition was to provide a baseline reference to account for any task-related changes in motor activity (e.g. heightened arousal). These reference trials also offered a baseline for normalizing MEP amplitudes in this study. The outline of testing events is displayed in Fig. 1B.

\subsubsection{Data processing and statistical analysis}

Any trials where the participant either (a) reached for the handle when it was covered, or (b) reached prior to the tone were excluded. Background EMG was determined from the root mean square of EMG activity in a time window of $100 \mathrm{~ms}$ immediately prior to TMS onset. If background EMG in this time window exceeded $10 \mu \mathrm{V}$ for a given muscle, the trial was discarded. Moreover, any trials where a muscle produced a very small MEP amplitude (i.e. $<100 \mathrm{mV}$ peak-to-peak) were excluded. Finally, outliers were identified as those values falling outside the threshold defined by 1.5 times the interquartile range, and these outliers were excluded from further analysis.

MEP amplitude was determined as the rectified EMG area beginning at the positive EMG signal deflection for each hand muscle, and ending $50 \mathrm{~ms}$ post TMS. The use of rectified EMG area to assess MEP amplitude is similar to methods by Franca et al. [6]. While Franca and colleagues did not specify a time interval, Hasbroucq et al., used a set time window of $18 \mathrm{~ms}-40 \mathrm{~ms}$ post-TMS to capture the area of the rectified EMG signal [14]. To determine if this time window was suitable for our needs, we conducted preliminary analysis and found that by $\sim 50 \mathrm{~ms}$ the rectified MEP waveform had subsided back to baseline. MEP offsets were much more variable and harder to detect versus MEP onsets, therefore our approach was to use a common stop point for the analysis window (i.e. $50 \mathrm{~ms}$ ). Conversely, MEP onsets could be clearly detected for each trial, and each participant, thus we opted to use a specific onset. The time window of rectified MEP area was intended to optimally quantify the magnitude of the MEP signal for a given trial.

MEP amplitudes were converted into z-scores to reduce potential variability between test blocks within an individual, and to reduce inter-subject variability. This approach is consistent with previous TMS-based studies where MEP data is normalized to aid comparison $[14,15]$. The mean and standard deviations of the MEP amplitudes during 'no-vision' trials for each test block were used as a reference, for 
(A)

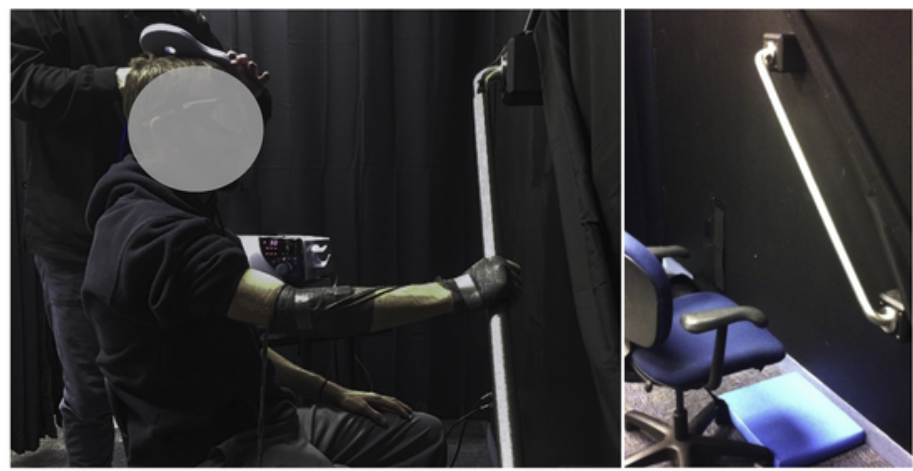

(B)

Motor-Evoked Potential

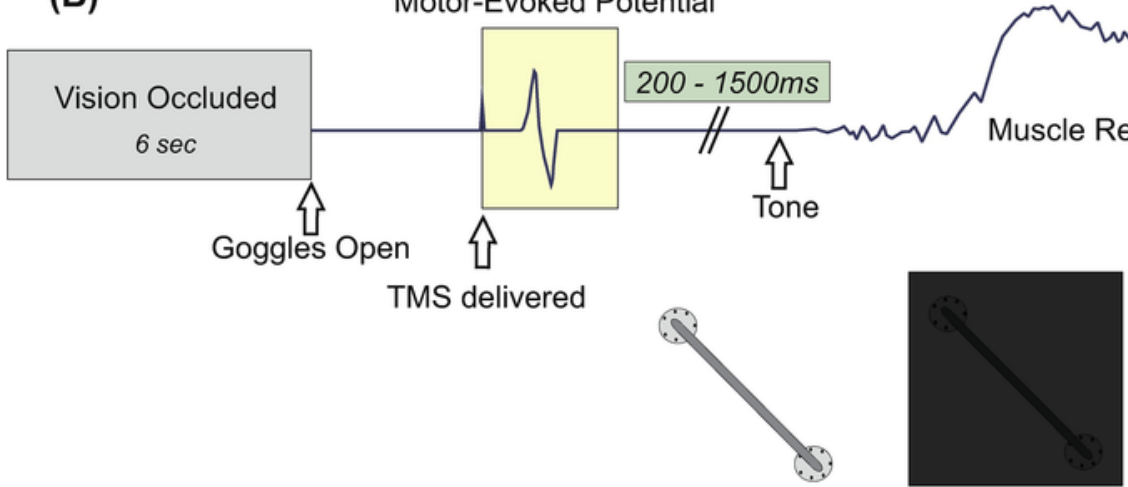

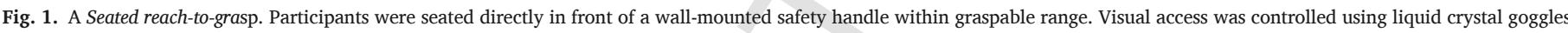

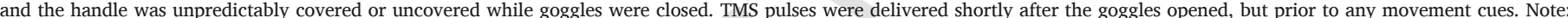

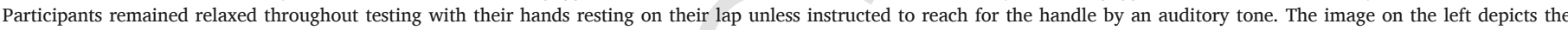

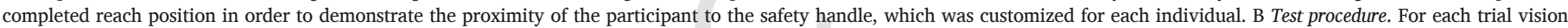

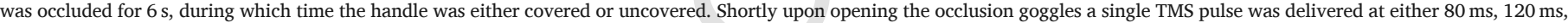
or $160 \mathrm{~ms}$ post-opening in separate test blocks. For some trials, auditory cues were presented after the goggles were opened (but after TMS delivery).

each participant separately. The individual MEP amplitudes observed in the handle and no-handle conditions were converted into z-scores calculated from this reference. The assumption in referencing all trials to a 'no-vision' baseline, is that the general state of the motor system will be accounted for in this reference value. These normalized values were subsequently grouped for statistical analysis. MEP analyses were limited to the intrinsic hand muscles (FDI, OP and ADM). Given the synergistic nature of FDI and $\mathrm{OP}$, these muscles were averaged together to assess grasping affordance. Conversely, the ADM was analyzed separately consistent with the distinct role of muscle when performing different grip types [16] and further consistent with the disparate influence of an affordance effect previously shown in FDI and OP versus ADM [6]. In the present study, the forearm muscle (ECR) was used to detect response onset as it provided a reliable indication of the initial arm response when reaching towards the handle. However, due to inconsistent MEPs in ECR (as the threshold was based on FDI), this muscle was not included in the MEP analysis, which instead focused on intrinsic hand muscles.

Two within-subject's factors are defined as 'Time' of TMS delivery $(80 \mathrm{~ms}, 120 \mathrm{~ms}, 160 \mathrm{~ms}$ ), and visual access to the 'Handle' (Handle, No-Handle), while 'Age' (Young, Old) is a between-subjects factor. The standard approach of a $2 \times 2 \times 3$ mixed or repeated-measures analysis of variance (RM ANOVA) was planned, however early tests for the assumption of homogeneity of variance reveled greater variance on MEP of the FDI-OP average among the younger participants, Leven's $F_{1,250}$ $=4.0475, \mathrm{p}=.04531$. Multilevel models (MLM) offer a more flexible framework to answer the same questions without relying on the same assumptions, thus a 2-level, random intercept MLM, with observations nested within subjects, was used to test the MEP of each muscle set separately (FDI-OP ${ }_{\text {average, }} \mathrm{ADM}$ ). Level one factors included 'Time' and 'Handle', and the level two factor was 'Age'. Furthermore, 'Handle' was allowed to moderate both 'Time' and 'Age' by incorporating a 3-way cross-level interaction. MLM also allow for pairwise planned (or post hoc) comparisons via estimated marginal means. A standard 5\% significance level was used throughout.

Based on our findings in the young adults [9], FDI-OP average $_{\text {was fa- }}$ cilitated when TMS was delivered specifically at $120 \mathrm{~ms}$ after viewing the handle, whereas ADM was reduced across time when the handle was present. Therefore, the planned comparisons here focus on testing if these effects are also evident in older adults.

\section{Results}

Behavioural responses revealed that young adults initiated their reach to handle (determined via EMG activity onset of the ECR muscle) on average $234 \mathrm{~ms}+/-50 \mathrm{~ms}$ following the imperative tone, whereas older adults initiated their reach significantly later, on average $292 \mathrm{~ms}$ $+/-108 \mathrm{~ms}\left(\mathrm{t}_{40}=-2.725, \mathrm{p}=.010\right)$. Older adults reached more often prior to the movement cue $\left(\mathrm{t}_{40}=-3.543, \mathrm{p}=.001\right)$, and reached more frequently when the handle was covered $\left(t_{40}=-2.406, p=.021\right)$ shown in Fig. 2.

Fig. 3A displays estimated marginal means for FDI-OP ${ }_{\text {average }}$ based on the MLM fit including the three-way (Time $\mathrm{x}$ Handle $\mathrm{x}$ Age) interaction. Despite this interaction falling short of significance per the likelihood ratio test of nested models $\left(\chi^{2}{ }_{3}=4.297, \mathrm{p}=.231\right)$, two a priori contrasts were assessed using the Sidak method of adjustment [17]. 

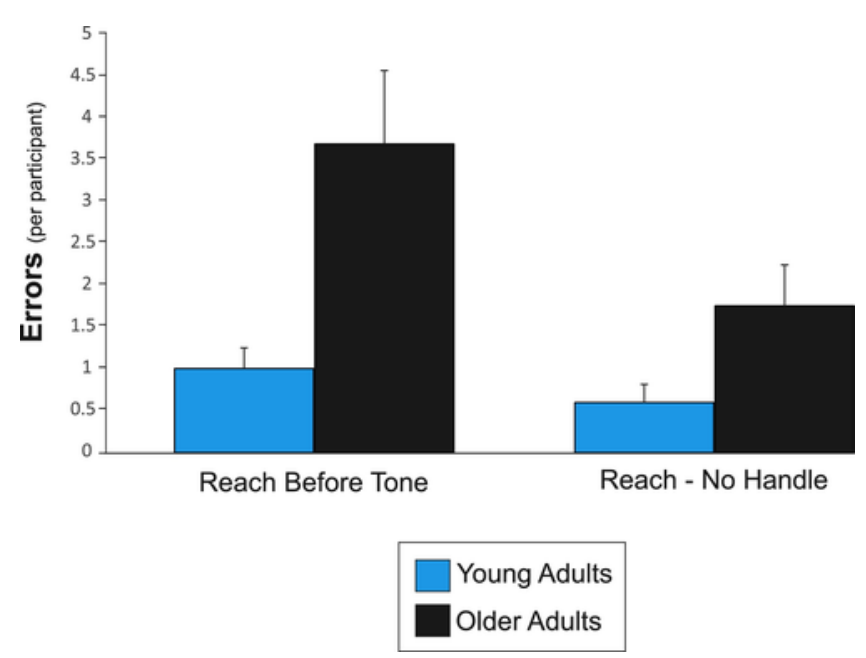

Fig. 2. Response Errors. This figure depicts errors for both age groups with the type of error divided into two groups - before the tone, or reaching when the handle is covered. Errors are presented as the average absolute number of errors per participant throughout testing.

The presence of a handle did in fact increase CSE for FDI-OP ${ }_{\text {average }}$ in the young at $120 \mathrm{~ms}\left(\mathrm{t}_{200}=2.717, \mathrm{p}=.014\right)$, but not the older adults $\left(\mathrm{t}_{200}=-0.272, \mathrm{p}>.954\right)$. Fig. 3B displays estimated marginal means for ADM for a similarly defined MLM. In the same way, both a priori pairwise comparisons were assessed using a Sidak method of adjustment. The presence of a handle did not have a significant effect among the older adults $\left(\mathrm{t}_{185}=0.277, \mathrm{p}>.953\right)$, nor the young $\left(\mathrm{t}_{185}=-0.992\right.$, $\mathrm{p}>.541)$ in ADM.

\section{Discussion}

The change in hand muscle activity that arose when young adults viewed a wall-mounted safety handle was absent in older adults. When young adults viewed this handle, the muscles involved in hand opposition (FDI and OP) were facilitated $120 \mathrm{~ms}$ after access to vision. In contrast to young adults, no modulation of CSE was noted for any muscle, at any time point when older adults viewed the handle. To the best of our knowledge this is the first study to show that priming hand responses when viewing graspable objects is attenuated in older adults.

Distinct patterns of cortical facilitation emerge for hand muscles prior to grasping either a small handle, or grasping a disk which requires a wider hand aperture [18], and these patterns correspond to the EMG activity during subsequent grasping of these different objects [16]. A selective pattern of CSE matching grasp type has been shown $150 \mathrm{~ms}$ after viewing an object, meaning that object-related information used to shape the hand reaches the motor cortex by this time [18]. In the study by Prabhu and colleagues (2007), no changes were detected in their earlier TMS probes ( $50 \mathrm{~ms}$ and $100 \mathrm{~ms}$ ), consistent with limits for how quickly visual information impacts frontal networks $[19,20]$. This is also in line with present results where no effects were detected when TMS was applied $80 \mathrm{~ms}$ upon viewing the handle. Franca and colleagues (2012) used TMS to measure changes in MEP amplitude when subjects viewed small objects that could be grasped using a precision grip, but without any requirement to move. They revealed facilitation in FDI and OP when TMS was delivered $120 \mathrm{~ms}$ post-vision (but not at $150 \mathrm{~ms}$ and $180 \mathrm{~ms}$ ) while ADM was unaffected by viewing these objects. This finding suggested that these hand muscles were selectively engaged to match the required precision grip. It is important to emphasize that the priming effect noted by Franca et al. (2012), was uncoupled from any need to act, supporting the notion that the visual world is automatically put into motor terms even without intention to move. In contrast to the findings by Franca et al,
(2012), Cattaneo and colleagues (2005) did not see facilitation when TMS was delivered after participants simply viewed graspable objects [16]. However, their probe into the dynamics of cortical excitability was much later (i.e. $1200 \mathrm{~ms}$ vs. $120 \mathrm{~ms}$ ) than when the effects were noted by Franca et al. (2012) or in present results. Collectively, this may indicate that visual priming for hand action quickly fades or may even be actively suppressed until immediately prior to movement onset. The absence of continued facilitation throughout the delay period may reflect impulse control during a delay period where the planned action is withheld until required [21].

The previous analysis in our lab focused exclusively on the young adults and revealed reduced ADM activation when viewing the handle [9]. This contrasts the increase in FDI and OP, both muscles which act to close the hand. The decrease in $\mathrm{ADM}$ when viewing the handle was originally interpreted to reflect the role of ADM in abducting the little finger (i.e. antagonist to hand opposition), thereby suppressing an antagonist for hand closure. Visual inspection of Fig. 3B does suggest that ADM is indeed lower across time points when viewing the handle in the young adults. However, when compared across age groups this effect was no longer statistically significant using the MLM, perhaps reflecting insufficient power to detect a difference with this model.

Recent evidence highlights the importance of cognitive resources in visual priming of action, which suggests a possible reason for its absence in older adults when viewing the safety handle. Freeman and colleagues (2016) demonstrated that a higher working memory load abolished the motor affordance effect in young adults [10]. In particular, their results showed that the higher cognitive burden resulted in a tonic suppression of motor output, thus interfering with expression of CSE changes related to vision. Given age-related declines in cognitive function [11], one implication of present results is that older adults may have a reduced capability for preparing motor networks when viewing objects. It is possible that networks upstream of the primary motor cortex may continue to form motor associations when viewing graspable objects, but that these latent motor representations are unable to be expressed through an inhibited motor cortex. It should also be recognized that aging impacts the entire visual pathway ranging from diseases affecting the eye itself (e.g. cataracts) up to changes in visual cortices [22-24]. Therefore, we can't entirely rule out direct age-related changes in the visual system that may contribute to the present loss of motor priming. In fact, older adults tend to recruit more cortical regions such as prefrontal cortex, and engage occipital cortex less efficiently during visual matching tasks compared with young adults [24]. These results suggest that processing visual information (at least during attention demanding tasks) requires more cognitive resources in older adults. Regardless of where in the chain visual priming for action is impeded, an impaired ability to engage relevant motor networks when viewing our surroundings could reasonably be expected to compromise behavior.

Support handles are increasingly relied upon to provide stability and ensure safety as we age. This includes the use of handles to transition between sitting and standing postures, and handrails to climb stairs. Support handles can also help us regain stability following an unexpected loss of balance. Several studies have shown that compensatory reach-to-grasp reactions play a significant role when recovering from postural perturbation [25-29]. The fact that the cerebral cortex generates the early hand reaction to quickly grasp a supportive handle $[27,26]$ suggests that in the same way that visual priming shapes voluntary grasping behavior, this mechanism may likewise bias balance recovery actions suited to our surroundings. If true, the fact that this priming could take place even before the need for such action would offer a significant speed of response advantage. One theoretical implication from present results is that older adults may be deficient in such predictive grasp preparation, and thus lose the speed advantage when generating a compensatory arm reaction to grasp a stable handhold. 


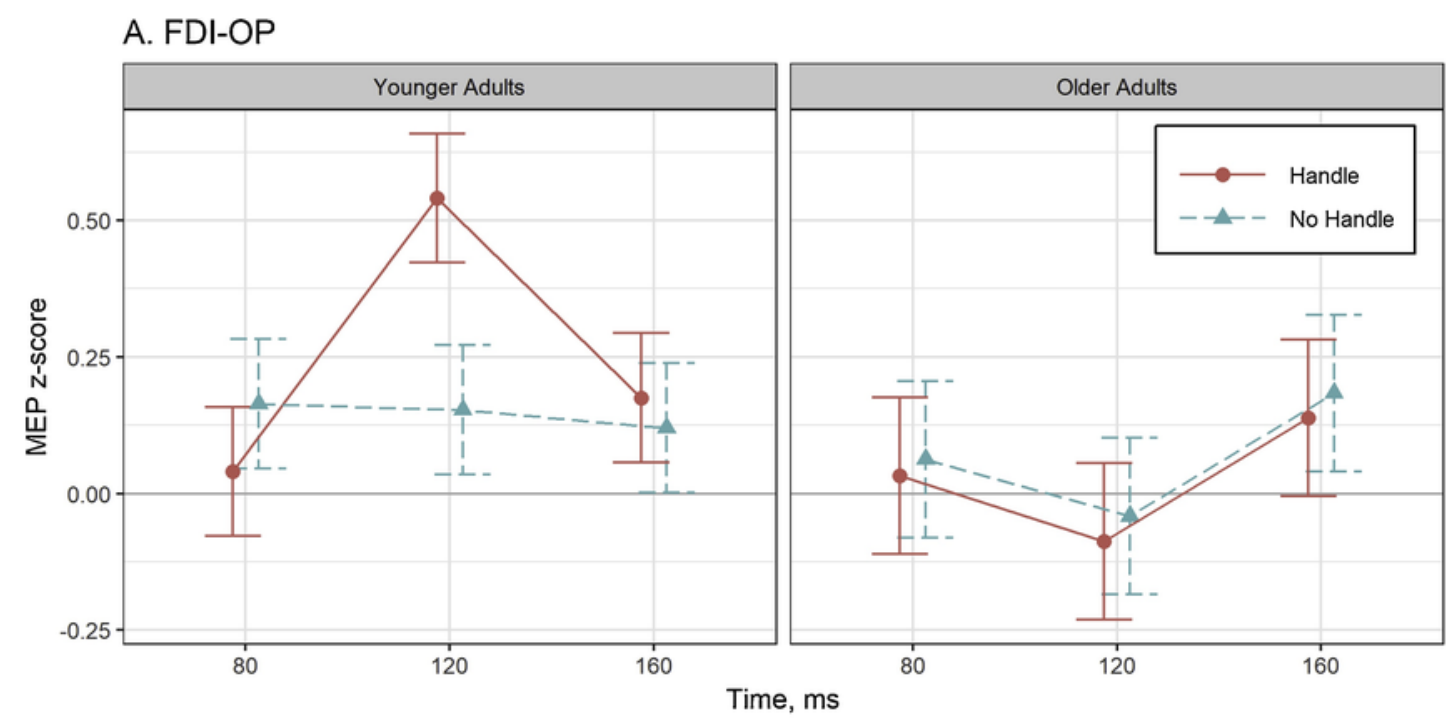

\section{B. ADM}
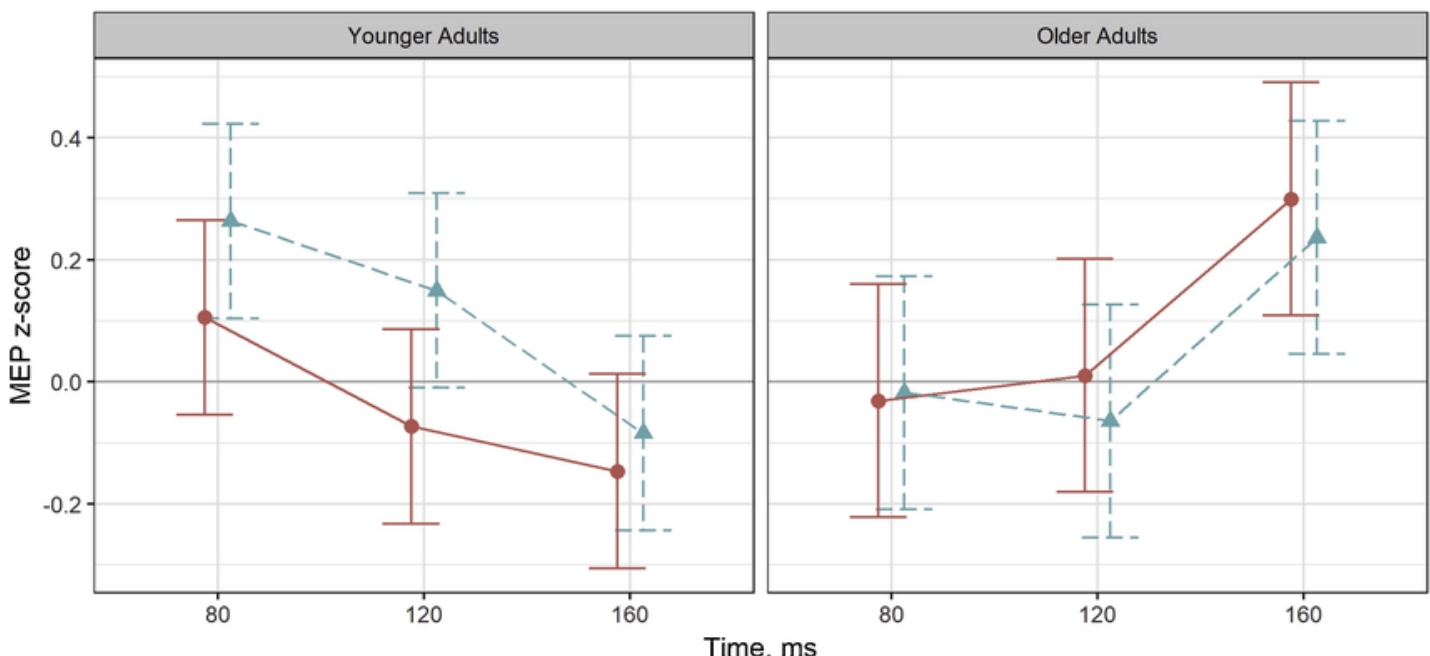

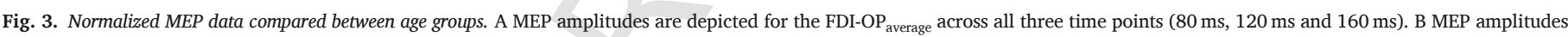

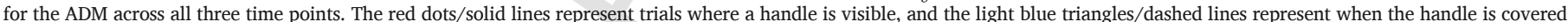

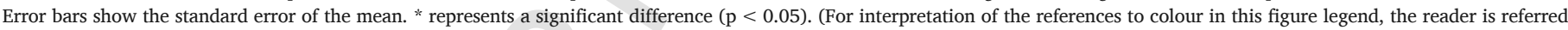
to the web version of this article).

\subsection{Methodological considerations}

A reactive reach-to-grasp task was used to focus participant attention directly onto the safety handle to help expose changes in CSE when viewing the handle. While the reaching behavior itself was unrelated to the present research question, it did provide an opportunity to verify changes one would expect in an aging motor system when performing a speeded 'go/no-go' test. Specifically, older adults responded more slowly and produced more reaching errors, consistent with age-related delays in processing speed [30-32] and inhibitory control deficits [33-35] respectively. The present study was not designed to detect a direct link between visual priming of intrinsic hand muscles and overt grasping behavior since response speed and reaching errors were based on the wrist extensor muscle (i.e. ECR produced the earliest onset for this reaching task). Given that older adults failed to show the same affordance effect as found in young adults, future work should employ study designs that can be used to investigate the relationship between visual priming of motor networks and the eventual grasping behavior.
One limitation in the present study is the fact that TMS is limited to a very early timeframe after opening the goggles. Given slower processing speeds in the older adults it is possible that our early TMS probes are failing to capture visual priming for action in these participants. Rather than abolished altogether, priming may simply be delayed in the older adults. However, such a delay would still likely have adverse effects when attempting to use visual feedback from the environment to smoothly direct grasping behavior in a timely manner. Such delays may be particularly problematic when time pressure is involved.

\section{Conclusions}

Older adults fail to show visual priming of hand action that was clearly noted in young adults when viewing a wall-mounted safety handle. If indeed such priming contributes to efficient interaction with graspable objects in our immediate environment, this would suggest that older adults may have a specific deficit in this regard. A deficiency in visual priming for goal-directed action would be especially impactful in those situations where limited time is available to prepare and exe- 
cute a targeted grasp (e.g. grasping a safety handle when unexpectedly perturbed).

\section{Contributors}

D.A.E. Bolton contributed to research design, data collection, analysis, and manuscript preparation.

S.E. Schwartz contributed to statistical analysis and manuscript preparation.

M. Mansour contributed to data collection, analysis, and manuscript preparation.

G. Rydalch contributed to data collection, analysis, and manuscript preparation.

D.W. McDannald contributed to data collection, analysis, and manuscript preparation.

\section{Conflict of interest}

The authors declare that they have no conflict of interest.

\section{Funding}

The authors received no external funding for this work.

Ethical approval

This study involved the use of human subjects, and the work described has received approval from the Utah State University Institutional Review board, and has been carried out in accordance with the code of ethics of the World Medical Association (Declaration of Helsinki) for experiments involving humans.

Informed consent was obtained for experimentation with human subjects. The privacy rights of human subjects were observed.

\section{Provenance and peer review}

This article has undergone peer review.

\section{Research data (data sharing and collaboration)}

There are no linked research data sets for this paper. Data will be made available on request.

\section{Acknowledgements}

The authors wish to thank Casey McPherson, Dayun Jeon, Haley Hayes, Elizabeth Edwards, James McJimsey, Ahmed Gaballah, and Paul Roberts for their assistance in collecting data for the pilot study. We also wish to thank Dr. Eadric Bressel and Dr. Chris Dakin for providing helpful insights into the study design.

\section{References}

[1] S.T. Grafton, L. Fadiga, M.A. Arbib, G. Rizzolatti, Premotor cortex activation during observation and naming of familiar tools, NeuroImage6 (1997) 231-236, https: //doi.org/10.1006/nimg.1997.0293.

[2] G. Buccino, M. Sato, L. Cattaneo, F. Rodà, L. Riggio, Broken affordances, broken objects: a TMS study, Neuropsychologia47 (2009) 3074-3078, https://doi.org/10. 1016/j.neuropsychologia.2009.07.003.

[3] P. Cardellicchio, C. Sinigaglia, M. Costantini, The space of affordances: a TMS study, Neuropsychologia49 (2011) 1369-1372, https://doi.org/10.1016/j. neuropsychologia.2011.01.021.

[4] S. Makris, A.A. Hadar, K. Yarrow, Viewing objects and planning actions: on the potentiation of grasping behaviours by visual objects, Brain Cogn. 77 (2011) 257-264, https://doi.org/10.1016/j.bandc.2011.08.002.

[5] Makris, et al., Are object affordances fully automatic? A case of covert attention, Behav. Neurosci. 127 (2013) 797-802.

[6] M. Franca, L. Turella, R. Canto, N. Brunelli, L. Allione, N.G. Andreasi, M. Desantis, D. Marzoli, L. Fadiga, Corticospinal facilitation during observation of graspable objects: a transcranial magnetic stimulation study, PLoS One 7 (2012)e49025https:// doi.org/10.1371/journal.pone.0049025.
[7] M. Tucker, R. Ellis, On the Relation Between Seen Objects and Components of Potential Actions, 1998https://doi.org/10.1037/0096-1523.24.3.830.

[8] J.J. Gibson, The Ecological Approach To Visual Perception, Houghton Mifflin, Boston, 1979.

[9] D.W. McDannald, M. Mansour, G. Rydalch, D.A.E. Bolton, Motor affordance for grasping a safety handle, Neurosci. Lett. 683 (2018) 131-137, https://doi.org/10 1016/j.neulet.2018.05.040.

[10] S.M. Freeman, S. Itthipuripat, A.R. Aron, High working memory load increases intracortical inhibition in primary motor cortex and diminishes the motor affordance effect, J. Neurosci. 36 (2016) 5544-5555, https://doi.org/10.1523/JNEUROSCI. 0284-16.2016

[11] T.A. Salthouse, Selective review of cognitive aging, J. Int. Neuropsychol. Soc. JINS16 (2010) 754-760, https://doi.org/10.1017/S1355617710000706.

[12] S. Rossi, M. Hallett, P.M. Rossini, A. Pascual-Leone, Safety of TMS Consensus Group, Safety, ethical considerations, and application guidelines for the use of transcranial magnetic stimulation in clinical practice and research, Clin. Neurophysiol. Off. J. Int. Fed. Clin. Neurophysiol. 120 (2009) 2008-2039, https://doi. org/10.1016/j.clinph.2009.08.016.

[13] P.M. Rossini, S. Rossi, Transcranial magnetic stimulation: diagnostic, therapeutic and research potential, Neurology68 (2007) 484-488, doi:68/7/484 [pii].

[14] T. Hasbroucq, A. Osman, C.-A. Possamä, B. Burle, S. Carron, D. Dépy, S. Latour, I. Mouret, Cortico-spinal inhibition reflects time but not event preparation: neural mechanisms of preparation dissociated by transcranial magnetic stimulation, Acta Psychol. (Amst.) 101 (1999) 243-266, https://doi.org/10.1016/ S0001-6918(99)00007-4.

[15] M.C. Klein-Flügge, S. Bestmann, Time-dependent changes in human corticospinal excitability reveal value-based competition for action during decision processing, J. Neurosci. 32 (2012) 8373-8382, https://doi.org/10.1523/JNEUROSCI.0270-12. 2012

[16] L. Cattaneo, M. Voss, T. Brochier, G. Prabhu, D.M. Wolpert, R.N. Lemon, A cortico-cortical mechanism mediating object-driven grasp in humans, Proc. Natl. Acad. Sci. U. S. A. 102 (2005) 898-903, https://doi.org/10.1073/pnas. 0409182102.

[17] B.H. Cohen, Explaining Psychological Statistics, 4th ed., Wiley, 2013.

[18] Prabhu Gita, Voss Martin, Brochier Thomas, Cattaneo Luigi, Haggard Patrick, Lemon Roger, Excitability of human motor cortex inputs prior to grasp, J. Physiol. (Paris) 581 (2007) 189-201, https://doi.org/10.1113/jphysiol.2006.123356.

[19] N.D. Schluter, M.F. Rushworth, R.E. Passingham, K.R. Mills, Temporary interference in human lateral premotor cortex suggests dominance for the selection of movements. A study using transcranial magnetic stimulation, Brain121 (1998) 785-799, https://doi.org/10.1093/brain/121.5.785.

[20] Y. Terao, H. Fukuda, Y. Ugawa, O. Hikosaka, R. Hanajima, T. Furubayashi, K. Sakai, S. Miyauchi, Y. Sasaki, I. Kanazawa, Visualization of the information flow through human oculomotor cortical regions by transcranial magnetic stimulation, J. Neurophysiol. 80 (1998) 936-946, https://doi.org/10.1152/jn.1998.80.2.936.

[21] S. Bestmann, J. Duque, Transcranial magnetic stimulation: decomposing the processes underlying action preparation, Neuroscientist 22 (2016) 392-405, https: //doi.org/10.1177/1073858415592594.

[22] N. Congdon, B. O'Colmain, C.C. Klaver, R. Klein, B. Muñoz, D.S. Friedman, J. Kem pen, H.R. Taylor, P. Mitchell, Causes and prevalence of visual impairment among adults in the United States, Arch. Ophthalmol. Chic. Ill 1960122 (2004) 477-485, https://doi.org/10.1001/archopht.122.4.477.

[23] A.M. McKendrick, Y.M. Chan, B.N. Nguyen, Spatial vision in older adults: perceptual changes and neural bases, Ophthalmic Physiol. Opt. 38 (2018) 363-375, https: //doi.org/10.1111/opo.12565.

[24] C.L. Grady, J.M. Maisog, B. Horwitz, L.G. Ungerleider, M.J. Mentis, J.A. Salerno, P. Pietrini, E. Wagner, J.V. Haxby, Age-related changes in cortical blood flow activation during visual processing of faces and location, J. Neurosci. 14 (1994) 1450-1462, https://doi.org/10.1523/JNEUROSCI.14-03-01450.1994.

[25] S.B. Akram, V. Miyasike-daSilva, K.V. Ooteghem, W.E. McIlroy, Role of peripheral vision in rapid perturbation-evoked reach-to-grasp reactions, Exp. Brain Res. 229 (2013) 609-619, https://doi.org/10.1007/s00221-013-3624-z.

[26] D.A. Bolton, R. Patel, W.R. Staines, W.E. McIlroy, Transient inhibition of primary motor cortex suppresses hand muscle responses during a reactive reach to grasp, Neurosci. Lett. 504 (2011) 83-87, https://doi.org/10.1016/j.neulet.2011.09.001.

[27] W.H. Gage, K.F. Zabjek, S.W. Hill, W.E. McIlroy, Parallels in control of voluntary and perturbation-evoked reach-to-grasp movements: EMG and kinematics, Exp. Brain Res. Hirnforsch. Cerebrale. 181 (2007) 627-637, https://doi.org/10.1007/ s00221-007-0959-3.

[28] E.C. King, S.M. McKay, K.C. Cheng, B.E. Maki, The use of peripheral vision to guide perturbation-evoked reach-to-grasp balance-recovery reactions, Exp. Brain Res. 207 (2010) 105-118, https://doi.org/10.1007/s00221-010-2434-9.

[29] K. Van Ooteghem, B. Lakhani, S. Akram, V. Miyasike Da Silva, W.E. McIlroy, Time to disengage: holding an object influences the execution of rapid compensatory reach-to-grasp reactions for recovery from whole-body instability, Exp. Brain Res. 231 (2013) 191-199, https://doi.org/10.1007/s00221-013-3682-2.

[30] T.A. Salthouse, B.L. Somberg, Isolating the age deficit in speeded performance, J Gerontol. 37 (1982) 59-63, https://doi.org/10.1093/geronj/37.1.59.

[31] T.A. Salthouse, Attentional blocks are not responsible for age-related slowing, J. Gerontol. 48 (1993) P263-P270, https://doi.org/10.1093/geronj/48.6.P263.

[32] T.A. Salthouse, Aging and measures of processing speed, Biol. Psychol. 54 (2000) 35-54, https://doi.org/10.1016/S0301-0511(00)00052-1.

[33] R.L. West, An application of prefrontal cortex function theory to cognitive aging, Psychol. Bull. 120 (1996) 272-292.

[34] R. West, C. Alain, Age-related decline in inhibitory control contributes to the increased Stroop effect observed in older adults, Psychophysiology37 (2000) 179-189.

[35] R.G. Cohen, J.G. Nutt, F.B. Horak, Errors in postural preparation lead to increased 